EESTI NSV TEADUSTE AKADEEMIA TOIMETISED. XV KÖIDE FOUSIKA-MATEMAATIKA-JA TEHNIKATEADUSTE SEERIA. 1966, NR. 2

ИЗВЕСТИЯ АКАДЕМИИ НАУК ЭСТОНСКОЙ ССР. ТОМ ХV СЕРИЯ ФИЗИКО-МАТЕМАТИЧЕСКИХ И ТЕХНИЧЕСКИХ НАУК. 1966, № 2

С. $У Л Ь М$

\title{
О ПОСТРОЕНИИ АЛГОРИФМОВ ДЛЯ ПРИБЛИЖЕННОГО РЕШЕНИЯ НЕКОТОРЫХ ЗАДАЧ ОПТИМАЛЬНОГО УПРАВЛЕНИЯ
}

Решение некоторых задач оптимального управления можно привести к задачам нахождения абсолютных минимумов некоторых функционалов в пространствах функций. Для приближенного решения последних можно использовать алгорифмы, выработанные для решения нелинейных уравнений. Так, в работах М. Аоки [1,2] для решения задач огтимального управления были обобщены метод Ньютона и некоторый вариант метода градиентов.

В настоящей статье на основании методов функционального анализа дается более общая схема построения методов такого типа для решения некоторых задач оптимального управления.

1. Пусть $\mathfrak{X}$ и $U$ - банаховы (гильбертовы) пространства; $\mathfrak{X}^{*}$ и $U^{*}-$ сопряженные к $⿱ 亠 乂 十 ्$ и $U$ пространства. Пусть оператор $K \in(U \rightarrow \mathfrak{X})$, а $c$ некоторый фиксированный элемент пространства $\mathfrak{x}$. Символ $(y, x)$ обозначает при фиксированном $y \in \mathfrak{X}$ * 3 начение линейного функционала в пространстве $\mathfrak{X}\left(\right.$ ср. [3]). Аналогичный смысл имеет $(v, u)$ при $v \ni U^{*}, u \in U$.

Рассмотрим функционал

$$
\varphi(u)=f(x, u)
$$

где $u \in U ; x=c+K u \in \mathfrak{X}$.

Введем обозначения *:

$F_{1}(x, u) \in \mathfrak{X}^{*}-$ частный градиент функционала $f(x, u)$ по $x$, т. е.

$$
f_{x}(x, u) k=\left(F_{1}(x, u), k\right) ; k \in \mathfrak{X} ;
$$

$F_{2}(x, u) \in U^{*}$ - частный градиент функционала $f(x, u)$ по $u$, т. е.

$$
\begin{aligned}
& f_{u}(x, u) h=\left(F_{2}(x ; u), h\right) ; h \in U ; \\
& \left.\begin{array}{l}
F_{1 x}(x, u) \\
F_{1 u}(x, u)
\end{array}\right\} \text { - частные производные оператора } F_{1}(x, u) \text {; } \\
& \left.\begin{array}{l}
F_{2 x}(x, u) \\
F_{2 u}(x, u)
\end{array}\right\} \text { - частные производные оператора } F_{2}(x, u) \text {. }
\end{aligned}
$$

* Символы $f_{x}(x, u), f_{u}(x, u)$ обозначают частные производные функционала $f(x, u)$. 
Тогда по определению (см., напр., [3])

$$
\begin{aligned}
\varphi^{\prime}(u) h & =\lim _{t \rightarrow 0} \frac{\varphi(u+t h)-\varphi(u)}{t}= \\
& =\lim _{t \rightarrow 0} \frac{f(c+K(u+t h), u+t h)-f(c+K u, u)}{t}= \\
& =\lim _{t \rightarrow 0} \frac{\left(F_{1}(c+K u, u), t K h\right)+\left(F_{2}(c+K u, u), t h\right)+\ldots}{t}= \\
& =\left(K^{*} F_{1}(c+K u, u)+F_{2}(c+K u, u), h\right)
\end{aligned}
$$

где $K^{*} \in\left(X^{*} \rightarrow U^{*}\right)-$ сопряженный к оператору $K$.

Итак, по (2)

$$
\operatorname{grad} \varphi(u)=\Phi(u)=K^{*} F_{\mathrm{I}}(c+K u, u)+F_{2}(c+K u, u):
$$

Дальше найдем:

$$
\begin{aligned}
\Phi^{\prime}(u) h=\lim _{t \rightarrow 0} & \left\{\frac{K^{*} F_{1}(c+K(u+t h), u+t h)-K^{*} F_{1}(c+K u, u)}{t}+\right. \\
& \left.+\frac{F_{2}(c+K(u+t h), u+t h)-F_{2}(c+K u, u)}{t}\right\}= \\
= & \lim _{t \rightarrow 0}\left\{\frac{K^{*} F_{1 x}(c+K u, u) t K h+K^{*} F_{1 u}(c+K u, u) t h+\ldots}{t}+\right. \\
& \left.+\frac{F_{2 x}(c+K u, u) t K h+F_{2 u}(c+K u, u) t h+\ldots}{t}\right\}= \\
= & \left(K^{*} F_{1 x} K+K^{*} F_{1 u}+F_{2 x} K+F_{2 u}\right) h,
\end{aligned}
$$

T. e.

$$
\begin{gathered}
\Phi^{\prime}(u)=K^{*} F_{1 x}(c+K u, u) K+K^{*} F_{1 u}(c+K u, u)+ \\
+F_{2 x}(c+K u, u) K+F_{2 u}(c+K u, u) .
\end{gathered}
$$

Отметим еще, что при фиксированном $u \in U$ сопряженный к линейному оператору $\Phi^{\prime}(u)$ есть оператор

$$
\Phi^{\prime *}(u)=K^{*} F_{1 x}^{*} K+F_{1 u}^{*} K+K^{*} F_{2 x}^{*}+F_{2 u}^{*},
$$

где $F_{1 x}^{*}, F_{1 u}^{*}, F_{2 x}^{*}, F_{2 u}^{*}$ - операторы, сопряженные соответственно к $F_{1 x}, F_{1 u}, F_{2 x}, F_{2 u}$.

2. Қритические точки функционала $\varphi(u)$ удовлетворяют уравнению

$$
\Phi(u)=0 .
$$

Фтметим, что если решение $u$ уравнения (5) единственно и выполне- 
ны достаточные условия минимума []ㅣ, то $u$ непременно является точкой абсолютного минимума функционала $\varphi(u)$.

Итак, для нахождения точек минимума (локальных или абсолютных) функционалов $\varphi(u)$ можно применить алгорифмы, используемые для решения нелинейного уравнения (5). Рассмотрим некоторые основные алгорифмы такого типа.

1. Градиентные методы (см., напр., $\left[{ }^{4-6}\right]$ )

a) $u_{n+1}=u_{n}-\varepsilon_{n} \Phi\left(u_{n}\right)$

ИЛИ

$$
u_{n+1}=u_{n}-\varepsilon_{n}\left[K * F_{1}\left(c+K u_{n}, u_{n}\right)+F_{2}\left(c+K u_{n}, u_{n}\right)\right]
$$

б) $u_{n+1}=u_{n}-\varepsilon_{n} \Phi^{\prime *}\left(u_{n}\right) \Phi\left(u_{n}\right)$

или

$$
\begin{gathered}
u_{n+1}=u_{n}-\varepsilon_{n}\left[K^{*} F_{1 x}^{*}\left(c+K u_{n}, u_{n}\right) K+F_{1 u}^{*}\left(c+K u_{n}, u_{n}\right) K+\right. \\
\left.+K^{*} F_{2 x}^{*}\left(c+K u_{n}, u_{n}\right)+F_{2 u}^{*}\left(c+K u_{n}, u_{n}\right)\right]\left[K^{*} F_{1}\left(c+K u_{n}, u_{n}\right)+\right. \\
\left.+F_{2}\left(c+K u_{n}, u_{n}\right)\right] .
\end{gathered}
$$

Здесь $\varepsilon_{n}$ - положительные числа, для выбора которых имеются различные возможности (см., напр., $\left[{ }^{5,6}\right]$ ).

3 а мечани е 1. Алгорифмы (6) и (7) в общем имеют смысл только тогда, когда $U=U^{*}$; в противном случае их надо заменить соответственно алгорифмами

и

$$
u_{n+1}=u_{n}-\varepsilon_{n} A \Phi\left(u_{n}\right)
$$

$$
u_{n+1}=u_{n}-\varepsilon_{n} B \Phi^{\prime *}\left(u_{n}\right) A \Phi\left(u_{n}\right) \text {, }
$$

где

$$
A, B \in\left(U^{*} \rightarrow U\right) \text {. }
$$

2. Методы Ньютона $\left[{ }^{7}\right]$

а) модифицированный метод:

$$
u_{n+1}=u_{n}-\left[\Phi^{\prime}\left(u_{0}\right)\right]^{-1} \Phi\left(u_{n}\right)
$$

или

$$
\begin{aligned}
u_{n+1}= & u_{n}-\left[K^{*} F_{1 x}\left(c+K u_{0}, u_{0}\right) K+K^{*} F_{1 u}\left(c+K u_{0}, u_{0}\right)+\right. \\
& \left.+F_{2 x}\left(c+K u_{0}, u_{0}\right) K+F_{2 u}\left(c+K u_{0}, u_{0}\right)\right]^{-1} \times \\
& \times\left[K^{*} F_{1}\left(c+K u_{n}, u_{n}\right)+F_{2}\left(c+K u_{n}, u_{n}\right)\right]
\end{aligned}
$$

б) основной метод:

$$
u_{n+1}=u_{n}-\left[\Phi^{\prime}\left(u_{n}\right)\right]^{-1} \Phi\left(u_{n}\right)
$$

или

$$
\begin{aligned}
u_{n+1}= & u_{n}-\left[K^{*} F_{1 x}\left(c+K u_{n}, u_{n}\right) K+K^{*} F_{1 u}\left(c+K u_{n}, u_{n}\right)+\right. \\
+ & \left.F_{2 x}\left(c+K u_{n}, u_{n}\right) K+F_{2 u}\left(c+K u_{n}, u_{n}\right)\right]^{-1} \times \\
& \times\left[K^{*} F_{1}\left(c+K u_{n}, u_{n}\right)+F_{2}\left(c+K u_{n}, u_{n}\right)\right] .
\end{aligned}
$$


3. Другой возможностью построения алгорифмов для решения уравнения (5) является использование обобщенных разделенных разностей.

Рассмотрим оператор

$$
\Phi(u)=K^{*} F_{1}(c+K u, u)+F_{2}(c+K u, u) .
$$

Обобщенная разделенная разность $\Phi\left(u^{\prime}, u^{\prime \prime}\right)$ определяется следующим образом (ср. [10]) :

а) для каждых фиксированных $u^{\prime}, u^{\prime \prime} \in U$ оператор $\Phi\left(u^{\prime}, u^{\prime \prime}\right)$ является линейным, причем

$$
\Phi\left(u^{\prime}, u^{\prime \prime}\right)\left(u^{\prime}-u^{\prime \prime}\right)=\Phi\left(u^{\prime}\right)-\Phi\left(u^{\prime \prime}\right) \text {; }
$$

б) $\Phi(u, u)=\Phi^{\prime}(u)$.

Аналогичным образом определяем частные разделенные разности для оператора $F(x, u)$ :

$$
\begin{aligned}
& F\left(x^{\prime}, x^{\prime \prime} ; u\right)\left(x^{\prime}-x^{\prime \prime}\right)=F\left(x^{\prime}, u\right)-F\left(x^{\prime \prime}, u\right) \\
& F(x, x ; u)=F_{x}(x, u)
\end{aligned}
$$

и

$$
\begin{aligned}
& F\left(x ; u^{\prime}, u^{\prime \prime}\right)\left(u^{\prime}-u^{\prime \prime}\right)=F\left(x, u^{\prime}\right)-F\left(x, u^{\prime \prime}\right) \\
& F(x ; u, u)=F_{u}(x, u) .
\end{aligned}
$$

Найдем выражение для оператора Ф( $\left.u^{\prime}, u^{\prime \prime}\right)$ :

$\Phi\left(u^{\prime}, u^{\prime \prime}\right)\left(u^{\prime}-u^{\prime \prime}\right)=\Phi\left(u^{\prime}\right)-\Phi\left(u^{\prime \prime}\right)=$

$=K^{*} F_{1}\left(c+K u^{\prime}, u^{\prime}\right)-K^{*} F_{1}\left(c+K u^{\prime \prime}, u^{\prime \prime}\right)+F_{2}\left(c+K u^{\prime}, u^{\prime}\right)-$

$-F_{2}\left(c+K u^{\prime \prime}, u^{\prime \prime}\right)=$

$=K^{*} F_{1}\left(c+K u^{\prime}, u^{\prime}\right)-K^{*} F_{1}\left(c+K u^{\prime \prime}, u^{\prime}\right)+K^{*} F_{1}\left(c+K u^{\prime \prime}, u^{\prime}\right)-$

$-K^{*} F_{1}\left(c+K u^{\prime \prime}, u^{\prime \prime}\right)+F_{2}\left(c+K u^{\prime}, u^{\prime}\right)-F_{2}\left(c+K u^{\prime \prime}, u^{\prime}\right)+$

$+F_{2}\left(c+K u^{\prime \prime}, u^{\prime}\right)-F_{2}\left(c+K u^{\prime \prime}, u^{\prime \prime}\right)=$

$=\left[K^{*} F_{1}\left(c+K u^{\prime}, c+K u^{\prime \prime} ; u^{\prime}\right) K+K^{*} F_{1}\left(c+K u^{\prime \prime} ; u^{\prime}, u^{\prime \prime}\right)+\right.$

$\left.+F_{2}\left(c+K u^{\prime}, c+K u^{\prime \prime} ; u^{\prime}\right) K+F_{2}\left(c+K u^{\prime \prime} ; u^{\prime}, u^{\prime \prime}\right)\right]\left(u^{\prime}-u^{\prime \prime}\right)$,

T. e.

$$
\begin{aligned}
\Phi\left(u^{\prime}, u^{\prime \prime}\right)= & K^{*} F_{1}\left(c+K u^{\prime}, c+K u^{\prime \prime} ; u^{\prime}\right) K+K^{*} F_{1}\left(c+K u^{\prime \prime} ; u^{\prime}, u^{\prime \prime}\right)+ \\
& +F_{2}\left(c+K u^{\prime}, c+K u^{\prime \prime} ; u^{\prime}\right) K+F_{2}\left(c+K u^{\prime \prime} ; u^{\prime}, u^{\prime \prime}\right) .
\end{aligned}
$$

Используя полученное выражение, можно для решения уравнения (5) построить алгорифмы:

a) интерполяционны й аналог метода гр адиентов [9]:

$$
u_{n+1}=u_{n}-\varepsilon_{n} \Phi^{*}\left(u_{n}, u_{n-1}\right) \Phi\left(u_{n}\right)
$$

(Ф* $\left(u_{n}, u_{n-1}\right)$ - сопряженный $\mathrm{k} \Phi\left(u_{n}, u_{n-1}\right)$ оператор) 


$$
\begin{aligned}
& u_{n+1}=u_{n}-\varepsilon_{n}\left[K^{*} F_{1}^{*}\left(c+K u_{n}, c+K u_{n-1} ; u_{n}\right) K+\right. \\
& +F_{1}^{*}\left(c+K u_{n-1} ; u_{n}, u_{n-1}\right) K+K^{*} F_{2}^{*}\left(c+K u_{n}, c+K u_{n-1} ; u_{n}\right)+ \\
& \left.+F_{2}^{*}\left(c+K u_{n-1} ; u_{n}, u_{n-1}\right)\right]\left[K^{*} F_{1}\left(c+K u_{n}, u_{n}\right)+F_{2}\left(c+K u_{n}, u_{n}\right)\right]
\end{aligned}
$$

предполагается, что $U=U^{*}$ (ср. замечание 1$)$;

б) метод хорд $[8,10]$ :

$$
u_{n+1}=u_{n}-\left[\Phi\left(u_{n}, u_{n-1}\right)\right]^{-1} \Phi\left(u_{n}\right)
$$

или

$$
\begin{gathered}
u_{n+1}=u_{n}-\left[K^{*} F_{1}\left(c+K u_{n}, c+K u_{n-1} ; u_{n}\right) K+K^{*} F_{1}\left(c+K u_{n-1} ; u_{n}, u_{n-1}\right)+\right. \\
\left.+F_{2}\left(c+K u_{n}, c+K u_{n-1} ; u_{n}\right) K+F_{2}\left(c+K u_{n-1} ; u_{n}, u_{n-1}\right)\right]^{-1} \times \\
\times\left[K^{*} F_{1}\left(c+K u_{n}, u_{n}\right)+F_{2}\left(c+K u_{n}, u_{n}\right)\right]
\end{gathered}
$$

в) метод С теффенсена (с р. $\left.\left.{ }^{[11}\right]\right)$ :

भли

$$
\begin{gathered}
u_{n+1}=u_{n}-\left[\Phi\left(u_{n}, u_{n}-A \Phi\left(u_{n}\right)\right)\right]^{-1} \Phi\left(u_{n}\right), \\
A \in\left(U^{*} \rightarrow U\right)
\end{gathered}
$$

$$
\begin{gathered}
u_{n+1}=u_{n}-\left[K^{*} F_{1}\left(c+K u_{n}, c+K v_{n} ; u_{n}\right) K+\right. \\
+K^{*} F_{1}\left(c+K v_{n} ; u_{n}, v_{n}\right)+F_{2}\left(c+K u_{n}, c+K v_{n} ; u_{n}\right) K+ \\
\left.+F_{2}\left(c+K v_{n} ; u_{n}, v_{n}\right)\right]^{-1}\left[K^{*} F_{1}\left(c+K u_{n}, u_{n}\right)+F_{2}\left(c+K u_{n}, u_{n}\right)\right]
\end{gathered}
$$

тде

$$
v_{n}=u_{n}-A\left[K^{*} F_{1}\left(c+K u_{n}, u_{n}\right)+F_{2}\left(c+K u_{n}, u_{n}\right)\right]
$$

4. Рассмотрим задачу оптимального управления. Предположим, что требуется минимизировать по и интеграл

$$
f(x, u)=\int_{0}^{T} H(x, u) d t
$$

причем

$$
\frac{d x}{d t}=a x+b u ; \quad x(0)=x_{0}
$$

Допустим, что $a=a(t), b=b(t)$ - непрерывные функции и $H(x, u)$ обладает нужными дифференциальными свойствами; $T$ - фиксированное число.

Обозначим через $U(t, \tau)$ разрешающую функцию для уравнения $\frac{d x}{d t}=a x ;$ тогда из (17)

$$
x(t)=U(t, 0) x_{0}+\int_{0}^{t} U(t, \tau) b(\tau) u(\tau) d \tau=c+K u
$$


где

$$
\begin{aligned}
c & =c(t)=U(t, 0) x_{0} \\
K u & =K u(t)=\int_{0}^{t} U(t, \tau) b(\tau) u(\tau) d \tau .
\end{aligned}
$$

Если $a=$ const, то, например, $U(t, \tau)=e^{a(t-\tau)}$.

Итак, в данном случае

$$
\begin{aligned}
f(x, u) & =\int_{0}^{T} H\left(c(t)+\int_{0}^{t} U(t, \tau) b(\tau) u(\tau) d \tau, u(t)\right) d t= \\
& =f(c+K u, u)=\varphi(u) .
\end{aligned}
$$

Выбираем $\mathfrak{X}=U=L^{2}[0, T]$. Тогда $\mathfrak{X}=\mathfrak{X} * \quad U=U^{*}$. Отметим, что если $u(t) \in L^{2}, U(t, \tau)$ непрерывна по $t$ и интеграл

$$
\int_{0}^{T} \int_{0}^{T} U^{2}(t, \tau) b^{2}(\tau) d t d \tau
$$

является ограниченным, то и $x(t) \in L^{2}$ непрерывна.

Определим скалярное произведение обычным образом:

$$
(u, v)=\int_{0}^{T} u(t) v(t) d t .
$$

Тогда

$$
\begin{aligned}
(v, K u) & =\int_{0}^{T} v(t) d t \int_{0}^{t} U(t, \tau) b(\tau) u(\tau) d(\tau)= \\
& =\int_{0}^{T} b(\tau) u(\tau) d \tau \int_{\tau}^{T} U(t, \tau) v(t) d t= \\
& =\int_{0}^{T} b(t) u(t) d t \int_{t}^{T} U(\tau, t) v(\tau) d \tau=\left(u, K^{*} v\right),
\end{aligned}
$$

T. e,

$$
K^{*} v=b(t) \int_{t}^{T} U(\tau, t) v(\tau) d \tau
$$

Так как

$$
f(x, u)=\int_{0}^{T} H(x(t), u(t)) d t
$$


TO

$$
\begin{aligned}
& F_{1}=\frac{\partial H}{\partial x} ; \quad F_{2}=\frac{\partial H}{\partial u} ; \quad F_{1 x}=F_{1 x}^{*}=\frac{\partial^{2} H}{\partial x^{2}} \\
& F_{1 u}=F_{2 x}=F_{1 u}^{*}=F_{2 x}^{*}=\frac{\partial^{2} H}{\partial x \partial u} ; \quad F_{2 u}=\frac{\partial^{2} H}{\partial u^{2}} \\
& F_{1}\left(x^{\prime}, x^{\prime \prime} ; u\right)=F_{1}^{*}\left(x^{\prime}, x^{\prime \prime} ; u\right)=\frac{\left.\frac{\partial H}{\partial x}\right|_{\substack{x=x^{\prime} \\
u=u}}-\left.\frac{\partial H}{\partial x}\right|_{\begin{array}{c}
x=x^{\prime \prime} \\
u=u
\end{array}}}{x^{\prime}-x^{\prime \prime}} \\
& F_{2}\left(x^{\prime}, x^{\prime \prime} ; u\right)=F_{2}^{*}\left(x^{\prime}, x^{\prime \prime} ; u\right)=\frac{\left.\frac{\partial H}{\partial ! u}\right|_{\substack{x=x^{\prime} \\
u=u}}-\left.\frac{\partial H}{\partial u}\right|_{\substack{x=x^{\prime \prime} \\
u=u}}}{x^{\prime}-x^{\prime \prime}} \\
& F_{1}\left(x ; u^{\prime}, u^{\prime \prime}\right)=F_{1}^{*}\left(x ; u^{\prime}, u^{\prime \prime}\right)=\frac{\frac{\partial H}{\partial x}\left|\begin{array}{l}
x=x \\
u=u^{\prime}
\end{array}-\frac{\partial H}{\partial x}\right|_{\substack{x=x \\
u=u^{\prime \prime}}}}{u^{\prime}-u^{\prime \prime}} \\
& F_{2}\left(x ; u^{\prime}, u^{\prime \prime}\right)=F_{2}^{*}\left(x ; u^{\prime}, u^{\prime \prime}\right)=\frac{\left.\frac{\partial H}{\partial u}\right|_{\substack{x=x \\
u=u^{\prime}}}-\left.\frac{\partial H}{\partial ! u}\right|_{\substack{x=x \\
u=u^{\prime \prime}}}}{u^{\prime}-u^{\prime \prime}}
\end{aligned}
$$

5. Теперь на основании формул (6)-(9) и (11)-(14) легко получйть различные алгорифмы для приближенной минимизации функционала (21) (или для решения задачи (16)-(17)). Выпишем подробно некоторые из них. Используем для краткости обозначения:

$$
\frac{\partial H_{(n)}}{\partial u}=\left.\frac{\partial H}{\partial u}\right|_{\begin{array}{l}
x=x_{n} \\
u=u_{n}
\end{array}} ; \quad \frac{\partial H_{(n)}}{\partial x}=\left.\frac{\partial H}{\partial x}\right|_{\begin{array}{l}
x=x_{n} \\
u=u_{n}
\end{array}} \quad \text { иІ Т. Д., }
$$

где

$$
x_{n}=U(t, 0) x_{0}+\int_{0}^{t} U(t, \tau) b(\tau) u_{n}(\tau) d \tau
$$

Алг горифм (6):

$$
\begin{gathered}
u_{n+1}(t)=u_{n}(t)-\varepsilon_{n}\left[b(t) \int_{t}^{T} U(\tau, t) \frac{\partial H_{(n)}}{\partial x}(\tau) d \tau+\frac{\partial H_{(n)}}{\partial u}(t)\right] \\
n=0,1, \ldots
\end{gathered}
$$

Алгори фм (9):

$$
u_{n+1}(t)=u_{n}(t)+\Delta u_{n}(t)
$$

$\Delta u_{n}(t)$ определяется на каждом шагу из линейного интегрального уравнения 
174

C. УЛЬм

$$
\begin{gathered}
b(t) \int_{t}^{T} U(\tau, t)\left\{\frac{\partial^{2} H_{(n)}}{\partial x^{2}}(\tau) \int_{0}^{\tau} U(\tau, s) b(s) \Delta u_{n}(s) d s\right\} d \tau+ \\
+b(t) \int_{t}^{T} U(\tau, t) \frac{\partial^{2} H_{(n)}}{\partial x \partial u}(\tau) \Delta u_{n}(\tau) d \tau+\frac{\partial^{2} H_{(n)}}{\partial u \partial x}(t) \int_{0}^{t} U(t, \tau) b(\tau) \Delta u_{n}(\tau) d \tau+ \\
+\frac{\partial^{2} H_{(n)}}{\partial u^{2}}(t) \Delta u_{n}(t)=-b(t) \int_{t}^{T} U(\tau, t) \frac{\partial H_{(n)}}{\partial x}(\tau) d \tau-\frac{\partial H_{(n)}}{\partial u}(t)
\end{gathered}
$$

Отметим, что алгорифм (25) при спецнальном выборе $\varepsilon_{n}$ и алгорифм (26) получены в $\left[{ }^{1,2}\right]$ из некоторых других соображений.

Алгорифм (7):

где

$$
u_{n+1}(t)=u_{n}(t)-\varepsilon_{n} \Delta u_{n}(t)
$$

$$
\begin{gathered}
\Delta u_{n}(t)=b(t) \int_{t}^{T} U(\tau, t)\left[\frac{\partial 2 H_{(n)}}{\partial x^{2}}(\tau) \int_{0}^{\tau} U(\tau, s) b(s) g_{n}(s) d s+\right. \\
\left.+\frac{\partial^{2} H_{(n)}}{\partial u \partial x}(\tau) g_{n}(\tau)\right] d \tau+\frac{\partial^{2} H_{(n)}}{\partial x \partial u}(t) \int_{0}^{t} U(t, \tau) b(\tau) g_{n}(\tau) d \tau+\frac{\partial^{2} H_{(n)}}{\partial u^{2}}(t) g_{n}(t),
\end{gathered}
$$

a

$$
g_{n}(t)=b(t) \int_{t}^{T} U(\tau, t) \frac{\partial H_{(n)}}{\partial x}(\tau) d \tau+\frac{\partial H_{(n)}}{\partial u}(t)
$$

Выбнрая, например, в формуле (27)

$$
\varepsilon_{n}=\frac{\left\|\Phi\left(u_{n}\right)\right\|^{2}}{\left\|\Phi^{\prime *}\left(u_{n}\right) \Phi\left(u_{n}\right)\right\|^{2}}
$$

или

$$
\varepsilon_{n}=\frac{\int_{0}^{T} g_{n}^{2}(t) d t}{\int_{0}^{T} \Delta u_{n}^{2}(t) d t},
$$

получим итерационный метод с минимальными ошибками $\left[{ }^{12}\right]$.

Алгорнфм (13):

$$
u_{n+1}(t)=u_{n}(t)+\Delta u_{n}(t) \quad(n=1,2, \ldots),
$$

где $\Delta u_{n}(t)$ определяется нз уравнения (26), если в нем величнны

$$
\frac{\partial^{2} H_{(n)}}{\partial x^{2}}, \quad \frac{\partial^{2} H_{(n)}}{\partial x \partial u}, \quad \frac{\partial^{2} H_{(n)}}{\partial u \partial x}, \quad \frac{\partial^{2} H_{(n)}}{\partial u^{2}}
$$


заменить соответственно величинами

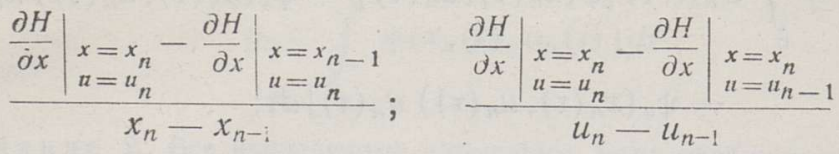

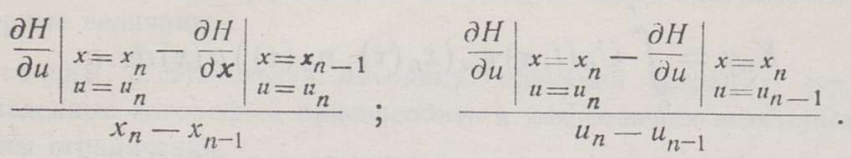

Отметнм, что для применения алгорифма требуются два начальных приближения.

Алг ори фм (10):

$$
u_{n+1}(t)=u_{n}(t)-\varepsilon_{n} \Delta u_{n}(t)
$$

где $\Delta u_{n}(t)$ вычисляется по формуле (27), если в ней величины (29) заменить соответственно величинами $(30)$.

6. Рассмотренные методы можно применить и для решения проблемы (ср. $\left.\left[{ }^{1,13}\right]\right)$ : минимизировать по и интеграл

$$
f(x, u)=\int_{0}^{T} H(x, u) d t
$$

причем

$$
\frac{d x}{d t}=\psi(x, u) ; \quad x(0)=x_{0}
$$

Пусть $u_{n}(t)$ - некоторое прнближение к оптимальному управлению; $x_{n}(t)$ - соответствующее решение уравнения (32).

Линеаризуем уравнение (32);

$$
\frac{d x}{d t}=\psi\left(x_{n}, u_{n}\right)+\psi_{x}\left(x_{n}, u_{n}\right)\left(x-x_{n}\right)+\psi_{u}\left(x_{n}, u_{n}\right)\left(u-u_{n}\right) .
$$

Из уравнения (33)

$$
\begin{gathered}
x(t)=U_{n}(t, 0) x_{0}+\int_{0}^{t} U_{n}(t, \tau)\left[\psi\left(x_{n}(\tau), u_{n}(\tau)\right)-\right. \\
\left.-\psi_{x}\left(x_{n}(\tau), u_{n}(\tau)\right) x_{n}(\tau)+\psi_{u}\left(x_{n}(\tau), u_{n}(\tau)\right)\left(u(\tau)-u_{n}(\tau)\right)\right] d \tau= \\
=c_{n}+K_{n} u,
\end{gathered}
$$

где

$U_{n}(t, \tau)$ - разрешающая функция для уравнения

$$
\frac{d x}{d t}=\psi_{x}\left(x_{n}, u_{n}\right) x
$$


$c_{n}=U_{n}(t, 0) x_{0}+\int_{0}^{t} U_{n}(t, \tau)\left[\psi\left(x_{n}(\tau), u_{n}(\tau)\right)-\psi_{x}\left(x_{n}(\tau) ; u_{n}(\tau)\right) x_{n}(\tau)-\right.$

$\left.-\psi_{u}\left(x_{n}(\tau), u_{n}(\tau)\right) u_{n}(\tau)\right] d \tau$

$$
K_{n} u=\int_{0}^{t} U_{n}(t, \tau) \psi_{u}\left(x_{n}(\tau), u_{n}(\tau)\right) u(\tau) d \tau
$$

Для огределения $u_{n+1}(t)$ используем один из алгорифмов, рассмотренных выше [т. е. осуществим один итерационный шаг для решения задачи (31), (33)]. Например, на основании алгорифма (7) получим

$$
u_{n+1}(t)=u_{n}(t)-\varepsilon_{n} \Delta u_{n}(t),
$$

где

$$
\begin{gathered}
\Delta u_{n}(t)=\left\{\psi _ { u } ( x _ { n } ( t ) , u _ { n } ( t ) ) \int _ { t } ^ { T } U _ { n } ( \tau , t ) \left[\frac{\partial^{2} H_{(n)}}{\partial x^{2}}(\tau) \int_{0}^{\tau} U_{n}(\tau, s) \psi_{u}\left(x_{n}(s), u_{n}(s)\right) X\right.\right. \\
\left.\times g_{n}(s) d s+\frac{\partial^{2} H_{(n)}}{\partial u \partial x}(\tau) g_{n}(\tau)\right] d \tau+\frac{\partial^{2} H_{(n)}}{\partial x \partial u}(t) \int_{0}^{t} U_{n}(t, \tau) \psi_{u}\left(x_{n}(\tau), u_{n}(\tau)\right) X \\
X g_{n}(\tau) d \tau+\frac{\partial^{2} H_{(n)}}{\partial u^{2}}(t) g_{n}(t)
\end{gathered}
$$

a

$g_{n}(t)=\psi_{u}\left(x_{n}(t), u_{n}(t)\right) \int_{t}^{T} U_{n}(\tau, t) \frac{\partial H_{(n)}}{\partial x}(\tau) d \tau+\frac{\partial H_{(n)}}{\partial u}(t) \quad(n=0,1,2, \ldots)$.

Индекс $(n)$ обозначает, что частные производные вычислены при значениях аргументов $x=c_{n}+K_{n} u_{n} ; u=u_{n}$.

Если $u_{n+1}(t)$ вычислен, то $x_{n+1}(t)$ найдем из уравнения $(32)$ и т. д.

Отметим, что вместо (33) можно использовать и другие способы линеаризации, например:

$$
\begin{aligned}
\frac{d x}{d t}= & \psi\left(x_{n}, u_{n}\right)-\frac{\psi\left(x_{n}, u_{n}\right)-\psi\left(x_{n-1}, u_{n}\right)}{x_{n}-x_{n-1}}\left(x-x_{n}\right) \\
& +\frac{\psi\left(x_{n-1}, u_{n}\right)-\psi\left(x_{n-1}, u_{n-1}\right)}{u_{n}-u_{n-1}}\left(u-u_{n}\right)
\end{aligned}
$$

или

$$
\begin{aligned}
\frac{d x}{d t}= & \psi\left(x_{n}, u_{n}\right)+\frac{\psi\left(x_{n}, u_{n}\right)-\psi\left(y_{n}, u_{n}\right)}{x_{n}-y_{n}}\left(x-x_{n}\right)+ \\
& +\frac{\psi\left(y_{n}, u_{n}\right)-\psi\left(y_{n}, u_{n-1}\right)}{u_{n}-u_{n-1}}\left(u-u_{n}\right)
\end{aligned}
$$


где

$$
y_{n}=\int_{0}^{t} \psi\left(x_{n}(t), u_{n}(t)\right) d t
$$

3 а меч а н и е 2. Все приведенные алгорифмы легко обобщаются на случай, если $x$ и $u$ векторные величины.

3 а ме ч а и е 3. Используя методику «функций штрафа» (ср., напр., [14, 15]), можно приведенные алгорифмы приспособить и для решения некоторых задач, где на $x$ и $и$ наложены ограничения.

3 амеч ани е 4. Для. исследования сходимости приведенных методов можно использовать общие теоремы о сходимости соответствующих методов для решения нелинейных операторных уравнений (ср., напр., [4-12]).

\section{Л И Т Е Р А У Р А}

1. A o k i M., J. Math. Anal. and Appl., 5, No. 3, 418-434 (1962).

2. A o k i M., J. Basic Engng, Trans. ASME, D 85, No. 2, 177-180 (1963).

3. В а й нб ер г М. М., Вариационные методы исследования нелинейных операторов, M., 1956.

4. В а й н б е р г М. М., Сибирск. матем. ж., 2, № 2, 201-220 (1961).

5. Л ин ьков Е. И., Уч. зап. Моск. обл. пед. ин-та, 96, $221-230$ (1960).

6. К и в и с тик Л. А., ДАН, 136, № 1, 22-25 (1961).

7. К а н то р о вич Л. В., Усп. матем. наук, 12 , вып. 6 (28), 89-195 (1948).

8. У ль м С., Изв. АН ЭССР. Сер. физ.-матем. и техн. наук, 13, № 3, $217-227$ (1964).

9. У ль м С., Изв. АН ЭССР. Сер. физ.-матем. и техн. наук, 12, № 2, 132-140 (1963).

10. С е р ге е в А. С., Сибирск. матем. ж., 2, № 2, 282-289 (1961).

11. У ль м. С., Ж. вычисл. матем. и матем. физ., 4, № 6, 1093-1097 (1964).

12. Фр и д м н В. М., ДАН, 139, № 5, 1063-1066 (1961).

13. B e $11 \mathrm{~m}$ a n R., Quart. Appl. Math., vol. 16, XVI, No. 3, 295-305 (1958).

14. ІШ а т р о в с к и й Л. И., Ж. вычнсл. матем. и матем. физ., 2, № 3, 488-491 (1962).

15. Фельд да ум А. А., Основы теории оптимальных автоматических систем, М., 1963.

\section{Ннститут кибернетики \\ Академии наук Эстонской ССР}

\section{Поступила в редакцию 14/VI 1965}

\section{S. ULM}

\section{ALGORITMIDE KOOSTAMISEST MÕNEDE OPTIMAALSE JUHTIMISE ULESANNETE LIGIKAUDSEKS LAHENDAMISEKS}

Artiklis esitatakse üldine, funktsionaalanalüüsil baseeruv metoodika mittelineaarsete vörrandite lahendamiseks kasutatavate algoritmide (Newtoni meetod, gradientide meetodid ning nende interpolatsıonıanaloogid) ülekandmiseks optimaalse juhtimise teatud tüüpi ülesannete ligikaudseks lahendamiseks.

\section{S. $U L M$}

\section{ON A TECHNIQUE OF THE CONSTRUCTION OF ALGORITHNS FOR APPROXIMATE EVALUATION OF SOME OPTIMUM CONTROL PROBLEMS}

This paper considers the solving of a certain class of optimum control problems, making use of general methods of the functional analysis (Newton's method, the methods of gradients and their interpolate analogues). 